\title{
FACTORES ASOCIADOS A LA SALUD Y EL BIENESTAR DEL ADULTO MAYOR: UN ESTUDIO DE CASOS EN LA PROVINCIA DE CIENFUEGOS, CUBA
}

Factors associated with the health and welfare of the elderly: A case study in the province of Cienfuegos, Cuba

\section{Caridad Luisa Casanova Rodríguez*}

Resumen: En este artículo ${ }^{1}$ se describen los resultados obtenidos, a partir de la aplicación de la última encuesta nacional de envejecimiento poblacional en el territorio de Cienfuegos, Cuba. Se analizan además algunos de los datos obtenidos en el territorio, con el fin de caracterizar socio-demográficamente a este grupo etareo, así como valorar algunos factores asociados a la salud y el bienestar, lo que permitirá un acercamiento de las tendencias de su comportamiento para el establecimiento y posterior aplicación de acciones psico-educativas, programas y medidas con fines predictivos, estén acorde con los lineamientos de la política económica y social del país. Es relevante lograr la inclusión de este

Profesora auxiliar e investigadora correspondiente al Centro de Estudios Socioculturales de la Facultad de Ciencias Sociales y Humanísticas de la Universidad “Carlos Rafael Rodríguez", Cuba. Email: casanova@ucf.edu.cu

1 Este trabajo cuenta con la coautoría de Elia Natividad Cabrera Álvarez, M. Sc, Máster en Administración de empresas. Profesora Auxiliar e Investigadora, Universidad "Carlos Rafael Rodríguez", Cuba. Email: elita@ucf.edu.cu

Elaine Isabel Cantero Tillet, M.Sc., especialista de la Oficina Territorial de Estadísticas de la Provincia de Cienfuegos, Cuba. Email: elaine@otecf.co.cu

Immer Ángel Ramos Reyes, profesor adjunto, Facultad de Humanidades de la Universidad Carlos Rafael Rodríguez, Cienfuegos, Cuba. Email: territorial.immer@otec.co.cu 
tipo de población como actores activos en la sostenibilidad y sustentabilidad de cada uno de los espacios territoriales de la Provincia de Cienfuegos.

Palabras claves: Envejecimiento, salud, bienestar, adulto mayor, política, Cienfuegos.

Abstract: A study of the results obtained, is from the application of the latest national survey of population aging in the territory of Cienfuegos, Cuba. It also discusses some of the data obtained in the territory with the goal of socio-demographically characterize this age group and to assess some factors associated with health and wellness, an approach allowing trends to their behavior the establishment and subsequent implementation of psycho-educational activities, programs and measures for predictive purposes, are consistent with the guidelines of the economic and social policy. It is relevant to achieve the inclusion of this population as active participants in sustainability and sustainability of each of the territorial areas of the province of Cienfuegos.

Keywords: Aging, health, welfare, elderly, politics.

\section{Introducción}

La población cubana actual muestra indicadores demográficos de países del primer mundo, bajos niveles de fecundidad desde finales de la década de los 80 del siglo Xx, bajos niveles de mortalidad, bajos y sostenidos valores de mortalidad infantil, a esto se suma el progresivo crecimiento de la esperanza de vida al nacer.

Es motivo de preocupación el notable incremento de la población de 60 años y más, tanto es así, que según investigaciones de la Oficina Nacional de Estadísticas e Información (ONEI) para 
1985 Cuba tenía un 11,3\% de personas de 60 años y más, y ya para el 2010 tenía un 17,8\%, lo cual la ubica en el Grupo III de Envejecimiento (mayor del 15\% la población de 60 años y más, respecto al total). En el término de 25 años, el envejecimiento se ha incrementado en 6,5 puntos porcentuales. La respuesta a esta problemática es el abordaje de este tema desde diferentes ciencias, no solo desde las ciencias médicas, sino también desde la demografía, la psicología, la geografía, la sociología y otras disciplinas. El punto de partida es comprender que la salud constituye un fenómeno transversal para toda la sociedad y, por tanto, debe ser tratado de forma multidisciplinar e integral, ya que sus impactos son irreversibles y resulta una fuente de preocupación para alcanzar una longevidad satisfactoria en la sociedad.

El proceso de envejecimiento se asocia con la denominada transición demográfica; en otras palabras, se trata de una evolución en la cual la reducción del tamaño de las cohortes de nacidos, implica un estrechamiento de la base de la pirámide inicial, lo que unido a la disminución de la mortalidad provoca un ensanchamiento, primero, en el centro de la misma y, segundo, en la cúspide. Esta disminución presenta varias etapas en Cuba, según estudios de la Comisión Económica para América Latina (CEPAL), la misma se encuentra en la última de estas etapas, con niveles muy bajos de fecundidad y mortalidad, aunque para algunos especialistas e investigadores ya se encuentra en una etapa post-transicional. (Alfonso, 2003).

Por las proyecciones de la población cubana y el crecimiento de la población que se enmarca en la tercera edad, se estima que aumentará la demanda de servicios de Geriatría, Gerontología, de Seguridad y Asistencia Social, siendo de vital importancia la evaluación de qué pasa, cómo viven, qué piensan, qué sienten, así como las condiciones de vida de estos grupos etareos.

En el período comprendido entre diciembre de 2010 y marzo de 2011 se aplicó la Encuesta Nacional de Envejecimiento Poblacional, 
en el marco de un proyecto con el Fondo de Población de Naciones Unidas (UNFPA), se hace con una muestra representativa conformada por personas de 60 años y más, residentes de forma permanente en las viviendas particulares del país, la cual fue diseñada por especialistas e investigadores del Centro de Estudios de Población y Desarrollo (CEPDE) de la Oficina Nacional de Estadística e Información (ONEI). La encuesta tuvo como objetivo fundamental investigar la situación de salud de los adultos mayores, su situación económica y social, así como la contribución que brindan las principales fuentes de apoyo a este grupo poblacional.

En el presente trabajo se realiza un estudio de casos tomando como base la encuesta de envejecimiento que se aplicó en la provincia de Cienfuegos, Cuba. Se investigaron 359 personas de 60 años y más. Se caracteriza socio-demográficamente a la población del territorio objeto de estudio; además se valoran algunos factores asociados a la salud y al bienestar de este grupo etareo. Los resultados obtenidos constituirán una herramienta para sustentar acciones, programas y medidas dirigidos en cada territorio e incrementar la sostenibilidad y la calidad de vida del adulto mayor.

\section{Desarrollo de la investigación}

El tema relacionado con el envejecimiento de la población de las sociedades industriales modernas constituye un tema cada vez más importante debido a las repercusiones que parece tener en la organización y estructura social, sobre todo en cuanto a las alteraciones del estado de bienestar que conllevan los cambios que se están produciendo actualmente.

En el 2009, las personas de 60 años y más conformaban el 17,4\% del total de la población y la edad media de la población cubana asciende a 38,10 años, lo que ubica al país entre los más envejecidos de América Latina y el Caribe. En el 2025, la población cubana debe alcanzar los 11134685 habitantes y liderará la región, con el 26,1\% de su población de adultos mayores. (ONE/CEPDE, 2010). 
Desde el punto de vista territorial, todas las provincias del país, excepto Guantánamo y el municipio especial de Isla de la Juventud se encuentran en el Grupo III con valores por encima de 15\%, siendo estos dos últimos territorios los que presentan los índices más bajos incluidos en el Grupo II, con valores entre el 10 y 15\% respectivamente que lo ubican como los territorios menos envejecidos del país.

La provincia de Cienfuegos no queda exenta del problema, el comportamiento de la población mayor de 60 años se expresa en un total de 70866 personas de ellos 34457 hombres y 36409 mujeres, lo que incide en el desarrollo de cada familia en que se encuentren ubicados y en cada uno de los propios sujetos mayores. (Casanova, 2011).

Se coincide con la afirmación de Philipp (2003) al decir que la familia es la institución más afectada por los cambios producidos por el así llamado "envejecimiento de la población” por múltiples motivos y en diversos aspectos; es la instancia que está en contacto permanente con la vejez a través de sus miembros. Tradicionalmente, el concepto de salud se medía mediante indicadores que recogían las pérdidas de la misma, como muerte o enfermedad, para cuya construcción se utilizaba la información proveniente de los servicios de salud y estadísticas vitales (INDEC, 2003). La ampliación de dicho concepto ha llevado a incorporar diferentes indicadores como ser el autoreporte o autopercepción de los individuos, enfermedades crónicas, limitaciones físicas, consultas realizadas en un período, etc.

En este trabajo se opta por valorar factores asociados a la salud y al bienestar de este grupo etareo, a través de la autopercepción del individuo y tomando en cuenta el estado general obtenido en la encuesta nacional aplicada. Se pretende contribuir al análisis del rol de la salud como una forma de capital humano, y el impacto de factores socioeconómicos, estilos de vida, etc. que inciden sobre la misma. A su vez, permite identificar la tendencia de este grupo 
poblacional, expuesto a riesgos de salud diferentes, los cuales implican demandas diferenciales de cuidados médicos.

\section{Características generales de la provincia de Cienfuegos}

La provincia de Cienfuegos se encuentra situada en el centro-sur del país. Limita al Norte con los municipios de Ranchuelo y Santo Domingo (provincia de Villa Clara) y con los municipios Calimete y Los Arabos (provincia de Matanzas); al este con el municipio de Manicaragua (provincia de Villa Clara) y con el municipio de Trinidad (provincia de Sancti Spíritus), al sur con el mar Caribe y al oeste con los municipios Ciénaga de Zapata y Calimete de la provincia de Matanzas. Tiene una extensión superficial de $4188,61 \mathrm{~km}^{2}$, por lo que es una de las cuatro más pequeñas del país, junto con La Habana, Artemisa y Mayabeque.

Al cierre del año 2010, Cienfuegos tenía una población de 407189 habitantes, con un $80,1 \%$ de la misma viviendo en zona urbana; el índice de masculinidad es de 1 132, la tasa de mortalidad infantil fue de 3,7 por cada mil nacidos vivos y posee una esperanza de vida al nacer de 78,28 años, la cual, al igual que la del país ha tenido años de ganancias en los últimos 50 años.

Un análisis de las pirámides de población elaborado con los datos de los censos de 1970 y 2002, muestra que los cambios en la estructura de la población que están ocurriendo en la provincia, el ensanchamiento en la base que existía inicialmente -además de la disminución de la mortalidad y la reducción de los cohortes de nacidos-, se ha ido trasladando hacia la cima. El segmento poblacional de 60 años y más va en aumento, resultando mayor la proporción de mujeres con respecto a los hombres, coincidiendo este comportamiento con las investigaciones realizadas a nivel nacional. 
Factores asociados a la salud y el bienestar del adulto mayor:

Un estudio de casos en la provincia de Cienfuegos, Cuba

\section{Estructura poblacional de Cienfuegos}

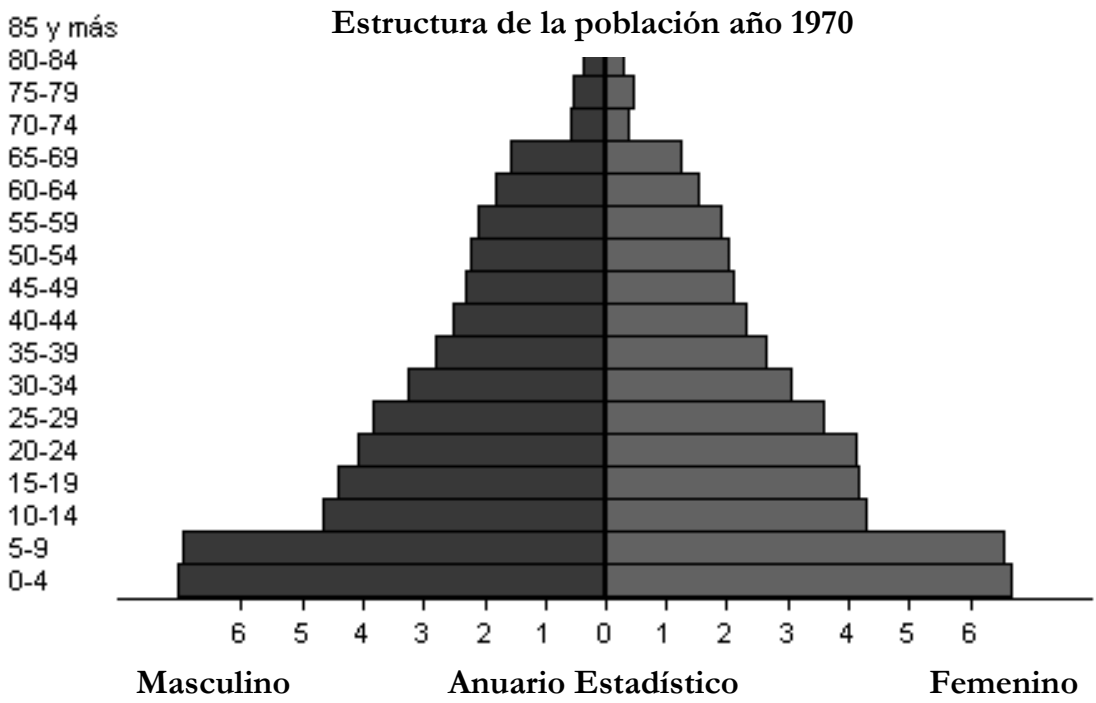

Estructura de la población año 2012

85 y más

$80-84$

$75-79$

$70-74$

$65-69$

$60-64$

$55-59$

$50-54$

$45-49$

$40-44$

$35-39$

$30-34$

$25-29$

20-24

15-19

10-14

5-9

$0-4$

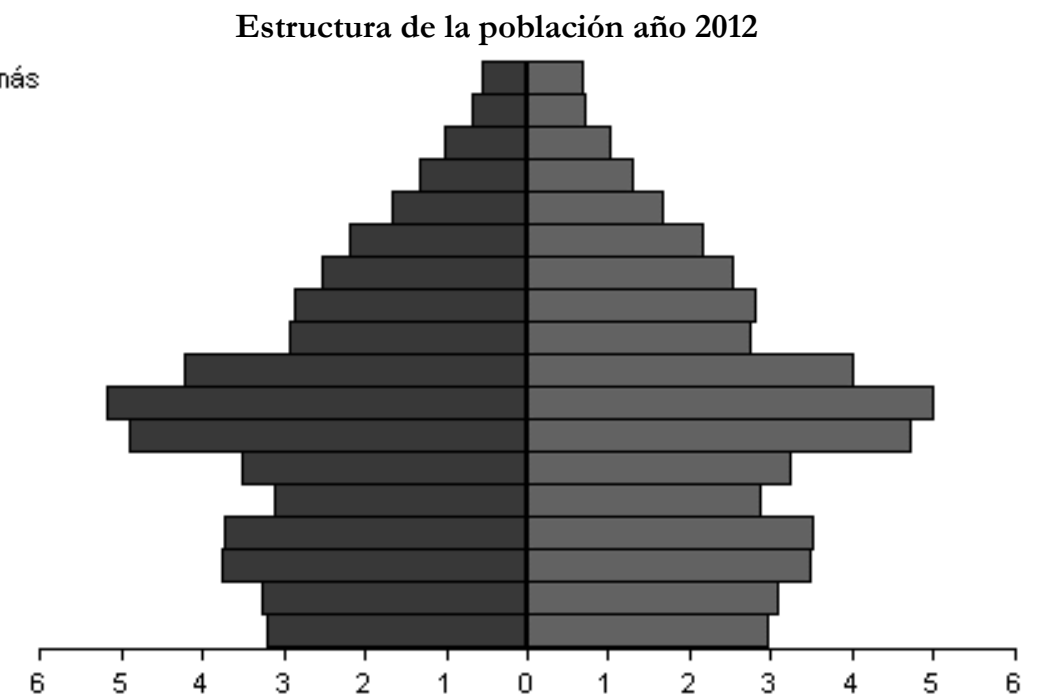

Masculino

Anuario Estadístico

Femenino

Fuente: ONEI. 
El proceso de envejecimiento que presenta el territorio es consecuencia principalmente de los bajos niveles de fecundidad que se vienen observando especialmente desde finales de los años 70 del pasado siglo, no obstante es importante destacar que Cuba fundamentalmente en las últimas décadas, se convierte en una sociedad con avances sustanciales y sostenibles en dimensiones básicas del desarrollo social, como son la educación, salud, salud reproductiva, seguridad y asistencia social, seguridad ciudadana y otros que en su interrelación han llevado a sus actuales y perspectivos niveles de envejecimiento.

Actualmente la Tasa Bruta de Reproducción (TBR) toma el valor de 0,85 hijas por mujer, de manera que al cierre del año 2010 la población de 0-14 años solo representaba el 17,3\% del total, mientras que la de 60 años y más se elevaba hasta $17,8 \%$, lo que en términos absolutos representa 72659 habitantes.

\section{Provincia Cienfuegos \\ Grado de Envejecimiento}
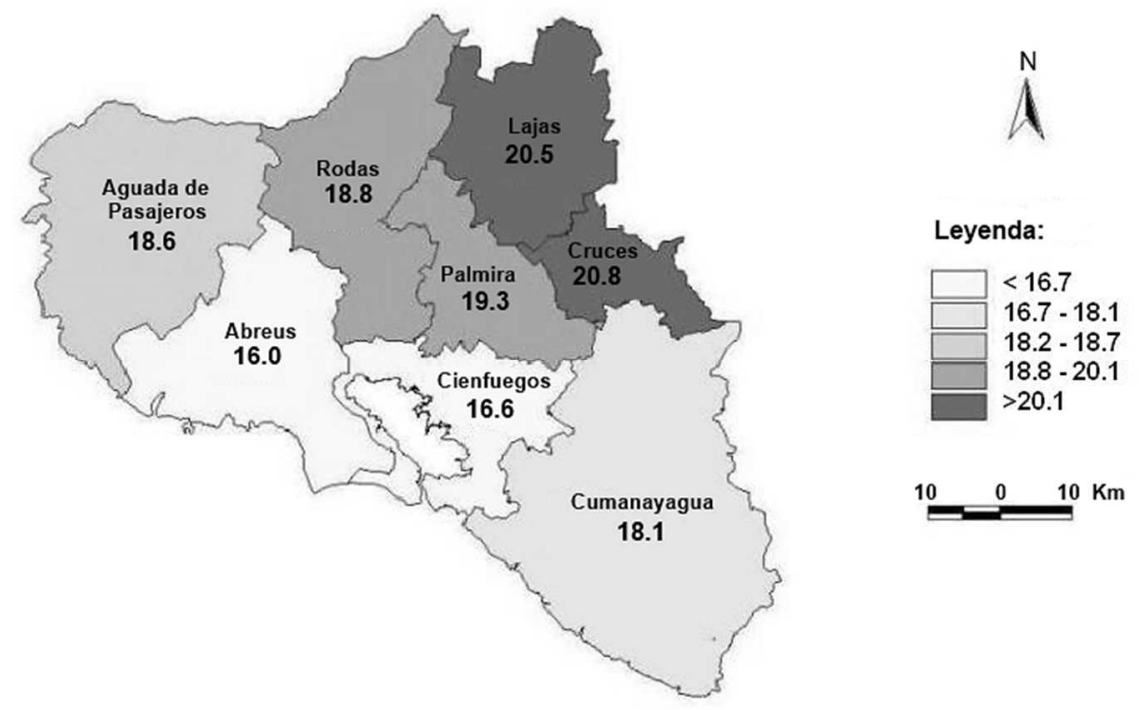

10

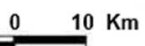

Fuente: ONEI. Cienfuegos 
En el mapa anterior se expresa el comportamiento del grado de envejecimiento en la provincia de Cienfuegos; los municipios del norte ocupan los valores más altos, $20,8 \%$ en Cruces y $20,5 \%$ en Lajas y los más bajos corresponden a los municipios del sur de la provincia, Abreus con el 16,0\% y Cienfuegos con 16,6\%.

\section{Selección de la muestra}

El método de muestreo se corresponde con un muestreo en dos fases. Esta técnica fue utilizada por primera vez por Neyman (1938) y es muy útil cuando existe poco o no existe ningún conocimiento a priori de la población. La muestra fue seleccionada, a partir de un Muestreo por Conglomerados Trietápico con estratificación de las unidades primarias de las viviendas con adultos mayores que estaban representadas en la encuesta provincial de Ocupación y Situación Económica de los Hogares (EPO) y la misma fue, a su vez, seleccionada del Diseño Muestral General (DMG) o Muestra Maestra elaborada en el período 2004-2005.

En las unidades muestrales de la primera etapa fueron seleccionadas 210 viviendas como promedio. Como unidades de segunda etapa se seleccionaron las áreas las cuales tienen 60 viviendas como mínimo. Las unidades de tercera y última etapa estuvieron constituidas por las secciones, con 5 viviendas como promedio. Las áreas fueron objeto de una selección sistemática con probabilidad proporcional al tamaño (PPT), tomando como medida de tamaño la cantidad de viviendas con residentes permanentes con que contaban las mismas. Las secciones se seleccionaron con probabilidades iguales $(\mathrm{PI})$.

El método de muestreo de la segunda fase se corresponde con un muestreo aleatorio simple estratificado, tomando como estratos los municipios y asentamientos de la provincia. Con este procedimiento de selección se garantiza una muestra auto-ponderada a nivel de estrato (provincia), lo cual significa que todos los individuos 
dentro del estrato tienen igual probabilidad final de selección en cada estrato.

El tamaño de la muestra fue determinado de manera tal que permitiera obtener resultados confiables de los principales indicadores de la encuesta a nivel regional por parte urbana y rural según sexo y tres grupos de edades (60 a 69; 70 a 79; 80 y más). Los principales indicadores tendrán un nivel de error inferior al $7 \%$ y una confiabilidad del $95 \%$, además se tuvo en cuenta un $20 \%$ de caída de muestra esperado para viviendas y personas. El tamaño teórico de la muestra asciende a 430 viviendas con adultos mayores. En cada vivienda fue entrevistado un adulto mayor.

En sentido general, el comportamiento de la muestra en la provincia, así como las causas de caídas de muestra se detallan a continuación:

\begin{tabular}{|l|c|c|c|c|c|}
\hline Provincia & $\begin{array}{c}\text { Muestra } \\
\text { Teórica } \\
\text { (Viv.) }\end{array}$ & $\begin{array}{c}\text { Muestra } \\
\text { real } \\
\text { (Viv.) }\end{array}$ & $\begin{array}{c}\text { Viviendas } \\
\text { entrevistadas }\end{array}$ & $\begin{array}{c}\text { Caída } \\
\text { Total }\end{array}$ & $\%$ \\
\hline Cienfuegos & 430 & 425 & 359 & 67 & 15.76 \\
\hline
\end{tabular}

Fuente: ONEI.

Nota: $\left(^{*}\right)$ El porciento de caída de muestra fue calculado, dividiendo para cada provincia la caída de viviendas por su total de viviendas a entrevistar o muestra real.

\section{Características sociodemográficas de la población objeto de estudio según la encuesta (ENEP-2010)}

Como se observa en la siguiente tabla, en Cienfuegos de los 359 casos investigados de 60 años y más, se concentra la mayor cantidad de personas de la tercera edad en el grupo de 80 años y más con un 24,8\%, seguido del 60-64 años con 22,3\% y el de 65-69 años con $22,6 \%$. 
Factores asociados a la salud y el bienestar del adulto mayor:

Un estudio de casos en la provincia de Cienfuegos, Cuba

\subsection{ESTRUCTURA DE LA POBLACIÓN OBJETO DE ESTUDIO POR GRUPOS DE EDADES}

\begin{tabular}{|c|c|c|}
\hline Grupos de Edades & Frecuencia & Porcentaje \\
\hline $60-64$ & 40 & 22.3 \\
\hline $65-69$ & 31 & 22.6 \\
\hline $70-74$ & 33 & 18.4 \\
\hline $75-79$ & 19 & 12.0 \\
\hline 80 y más & 32 & 24.8 \\
\hline Total & 359 & 100.0 \\
\hline
\end{tabular}

Fuente: BD Encuesta ENEP 2010. ONEI Cienfuegos

El comportamiento por sexo es bastante homogéneo, un 48.7\% de mujeres y el 51,3\% de hombres, dato este que se corresponde con la generalidad de este tipo de población en el país ya que la proporción en los hombres alcanza un $72,1 \%$ frente a un $68,9 \%$ en las mujeres.

Respecto al color de la piel el 18,1\% son negros y el $81,9 \%$ son blancos. El estado conyugal de los adultos mayores investigados muestra que el 46,0\% están casados o unidos, le siguen en proporción los viudos que son el $34,3 \%$, aspecto este que se corresponde con la bibliografía revisada, ya que son precisamente estas personas las que al perder a sus cónyuges o compañeros nutren la categoría de los viudos, disminuyendo el número de los divorciados o separados el 15,0\%, así como los que se declaran solteros (4,7\%). Se caracterizan por un nivel de escolaridad primario o bajo ya que pudiera interpretarse que se trata de un tipo de población que en su juventud no tuvieron facilidades para la instrucción media y superior (solo el 1,7\% cuenta con estudios universitarios) y que fueron expuestos a precarias condiciones económicas y sociales en que se desarrollaron sus vidas, pudiendo significar un obstáculo para priorizar el incremento de su nivel de escolaridad, frente a otras necesidades vitales. 


\section{Análisis descriptivo de los casos de estudio sobre el estado de salud, servicios, situación socioeconómica y el entorno, según la encuesta (ENEP-2010)}

Según las respuestas correspondientes a las categorías establecidas, se obtiene que la percepción de la salud vital, expresada por los adultos mayores es de promedio regular, identificando como enfermedades o padecimientos crónicos más recurrentes en estos adultos cienfuegueros, en primer lugar, la hipertensión arterial, que la padece el 51,3\% de los encuestados y en menor cuantía le siguen la artritis, reumatismo y artrosis que la padecen el 38,4\% y la cardiopatía isquémica o enfermedad coronaria en un 19,8\% de los encuestados. Estos padecimientos, según refieren los encuestados, limitan, en el rango regular, a 29,8\% y 15,9\% respectivamente, aunque el estado funcional lo evalúan como muy bueno, pudiéndose comprobar que más del 91,9\% de los adultos mayores realizan las actividades básicas de la vida diaria de forma independiente, teniendo en cuenta estos resultados. El 49,3\% de estos adultos, no sienten temor o incertidumbre por su salud en el futuro considerando las políticas sociales establecidas para su protección, así como las manifestaciones de la familia cubana en torno a este tipo de personas y debido a los logros y las configuraciones específicas que se ha adquirido en el país con una configuración de familias protectoras, donde el bienestar, permite a los sujetos una "compensación" de sus experiencias vitales, hecho que atañe asimismo al comportamiento en función del género de las personas mayores, muy particularmente en relación con su rol de abuelo o abuela en el seno familiar.

Fueron analizadas las actividades o servicios que los adultos mayores habían utilizado en los cuatro meses anteriores a la aplicación de la encuesta y el nivel de satisfacción con relación a los mismos, la encuesta arrojó que los servicios más utilizados son los servicios del médico de familia y la compra de medicamentos. Con respecto al servicio brindado por el médico de familia el 98.9\% se encuentran satisfechos. Situación semejante mostró el nivel de satisfacción 
con respecto a la compra de medicamentos con igual porciento de insatisfacción y ligero aumento en el nivel de satisfacción (46,2 \%). $\mathrm{Al}$ investigar la frecuencia con que los adultos mayores realizan actividades físicas como caminar, gimnasia, subir y bajar escaleras, limpiar y otras, pudo comprobarse que solo caminar (como actividad física) es realizada por $87,2 \%$ de los encuestados y al menos una vez por semana. Las restantes actividades de forma general, no la realizan. Es interesante destacar que este grupo de adultos mayores no se distinguen por participar en los círculos de abuelos, porque no lo desea ni la encuentra motivante, sino que se refleja una representación social de la instalación de exclusión familiar, prefiriendo las labores hogareñas y de cuidadores transmitidas socioculturalmente. La mayoría (79,9\%), participa en las actividades de las organizaciones de su comunidad (CDR, FMC, Asociación de combatientes, Núcleo zona) ${ }^{2}$, según informa el 79,9\% de ellos.

Del grupo de los encuestados, un $80 \%$ de los adultos se identifica como obrero o trabajador agropecuario, trabajador de los servicios y obrero no agropecuario.

Se encontró 220 jubilados, en una edad promedio de 60 años. En la actualidad, como promedio, los adultos mayores encuestados son jubilados o pensionados que no trabajan ni buscan trabajo, se mantienen trabajando un 19,5\%. De ellos, el 64,3\% plantea que la causa por la que se mantienen en activo es por sentirse útil y el $61,4 \%$ por aportar a la economía familiar, ya que con ello cubren los gastos fundamentales de la casa, alimentos y gastos médicos. El 75,2\% cuenta con la jubilación o pensión para el futuro, lo que ratifica su seguridad económica en el futuro.

Después de cumplir los 60 años solo iniciaron alguna actividad de trabajo cultural, artístico, social, turístico, económico o religioso; el $22 \%$ hizo este trabajo como algo que no había realizado antes.

2 Organizaciones políticas y sociales cubanas. 
Entre las actividades iniciadas en esta etapa se destacan, incorporarse a un nuevo trabajo y trabajar independientemente en un negocio propio/familiar favorecido por las nuevas políticas económicas del país, aunque las abuelas y los abuelos actuales siguen cumpliendo una función básica en cuanto al cuidado de nietos y nietas. Con esto prestan un apoyo importante a la familia, no solo en el caso de hacer posible en gran medida la introducción de las jóvenes madres en el mercado laboral, sino haciéndose cargo de tareas que son propias de las funciones del cuidado.

Entre las vías por las que los adultos mayores pueden obtener sus alimentos con mayor frecuencia, se encuentra la entrega regulada en establecimientos a bajos precios por parte del Estado (91,6\%, lo hace frecuentemente) y a través de mercados agropecuarios ubicados en la propia comunidad (44,6\% frecuentemente). La mayoría no obtiene alimentos por dietas alimenticias ni por enfermedad, ni por ayuda de vecinos o amistades, tampoco por compras en mercados en divisas, ni como apoyo recibido de alguna institución religiosa, ni sistema de atención a la familia.

Al indagar en los aspectos de la convivencia, el 79,1\% de los adultos mayores responde sentirse a gusto con las personas que con él conviven, ya sus criterios han sido valorados por sus familiares solo un $6,7 \%$ no está a gusto y el 14,2\% no responde a la pregunta y refieren que no han sido víctimas de situaciones de maltrato tales como comportamientos irrespetuosos, desatención, rechazo, privación de contactos sociales, abandono de sus parientes, así como por el uso ilegal o apropiación de su propiedad, de sus finanzas, del derecho al control de sus fondos personales o modificación de forzada de su testamento o engaños financieros. Muy concretamente y en consonancia con los datos que se han obtenido en el contexto internacional en estudios recientes sobre las abuelas y sus nuevas relaciones familiares, estas asumen cada vez más los valores de la autorrealización, independencia y autonomía personal en el trato con las nietas, los nietos y la familia. 


\section{Conclusiones}

Los resultados disponibles a partir de la investigación que se presenta en la provincia de Cienfuegos y otras fuentes de información estadística, permiten analizar una realidad que privilegiando la dimensión social del desarrollo presenta resultados relevantes en sus componentes.

A pesar de que la autopercepción de los individuos puede estar sujeta a sesgos, debido a problemas endógenos y de errores de medida, se encuentra que es un buen predictor de la mortalidad y, por ende, del estado de salud, incluyendo la evidencia del nivel socioeconómico como factor de riesgo para los más viejos siendo elementos de gran importancia para el desarrollo de estrategias preventivas de salud y para evaluar el impacto y la naturaleza de las desigualdades sobre la salud.

Se aportan datos que pudieran considerarse como tendencias de comportamientos de funcionabilidad, bienestar y salud en los adultos mayores de la provincia de Cienfuegos, constituyendo referentes predictivos de salud que refuerzan la necesidad de atenciones diferenciadas de estos valiosos actores sociales, en el establecimiento de políticas para el desarrollo local y la sostenibilidad del territorio, a partir de la búsqueda de espacios más comunes y armónicos donde las diferentes generaciones se involucren para su logro. 


\section{Referencias bibliográficas}

Alfonso Fraga, J. C. (2009). Envejecimiento y sociedad: El caso cubano. La Habana: Centro de Estudios de Población y Desarrollo.

Alfonso Fraga, J. C. (2009). Cuba: de la primera a la segunda transición demográfica. El descenso de la fecundidad". En la Fecundidad en América Latina ¿ Transición o Revolución? (pp. 331350). Santiago de Chile: Naciones Unidas.

Alfonso, J. C.; León, E. M.; Menéndez, J.; Marín, C. M.; Arcia, N., \& Martínez, L. (2005). Informe del Proyecto Salud, Bienestar y Envejecimiento de los adultos mayores en América Latina y el Caribe ( $S A B E)$. La Habana: Centro de Estudios de Población y Desarrollo; Centro Iberoamericano de la Tercera Edad.

Casanova Rodríguez, C. (2011). La autopercepción de la salud mental y la satisfacción vital del adulto mayor en la montaña y del longevo de procedencia rural en el territorio Cienfueguero. Universidady Sociedad, 3(3), 1-18.

Cuba. (2011). Anuario Estadístico Territorial. Cienfuegos: Oficina Nacional de Estadísticas e Información.

Cuba. (2011). División político administrativa. (Mapa Plegable. Escala 1:1 230 000). La Habana: Ediciones GEO.

Cuba. (2011). Informe Encuesta Nacional de Envejecimiento. Recuperado de http://www.one.cu

Jewell, R. T., Rossi, M., \& Triunfo, P. (2007). El estado de salud del adulto mayor en América Latina. Cuadernos de Economía 26(46), 147-167.

Organización Panamericana de la Salud. (1992). Informe final de la reunión de consulta sobre formulación de políticas de salud para los ancianos en América Latina y el Caribe [Serie informes técnicos N. $\left.{ }^{\circ} 24\right]$. (s. p.). 
Organización Panamericana de la Salud. (2001). Encuesta multicéntrica: Salud Bienestar y Envejecimiento (SABE) en América Latina y el Caribe. (Informe preliminar). Tegucigalpa: Organización Panamericana de la Salud.

Pereyra, A., Rossi, M., \& Triunfo, P. (2003). El gasto en cuidados médicos de las familias uruguayas. Trimestre Económico 277(1), 43-79.

Philipp, R. M. (2003). Transformaciones en el comportamiento en función del género en la vejez: El caso de las relaciones familiares actuales entre abuelas, abuelos y nietas y nietos. Revista de sociología, 70, 117-134. Recuperado de http://dialnet. unirioja.es/servlet/articulo?codigo $=836423$

Santellán, M. (2008). La salud a través de las encuestas de hogares en la Argentina. SEC informa [Boletín electrónico mensual] Recuperado de http://www.buenosaires.gob.ar/areas/hacien da/sis_estadistico/boletin/noviembre_08/notacentral.htm 


\section{Caridad Luisa Casanova Rodríguez, M. Sc.}

Es licenciada en Psicología y máster en Psicología Médica; profesora auxiliar; investigadora correspondiente al Centro de Estudios Socioculturales de la Facultad de Ciencias Sociales y Humanísticas de la Universidad "Carlos Rafael Rodríguez", provincia de Cienfuegos, Cuba. Es miembro de las juntas directivas de la Sociedad de Pedagogos de la provincia y también de la Sociedad de Pedagogos. Coordina proyectos a nivel institucional y territorial como internacional, relacionados con el tema del bienestar en diferentes grupos etáreos. La relevancia de sus investigaciones le ha permitido obtener diferentes premios y reconocimientos. Es autora de tres libros.

Email: casanova@ucf.edu.cu

Recibido: 19/02/2014 Aprobado: 14/06/2014 